\title{
Inconel-Steel Multilayers by Liquid Dispersed Metal Powder Bed Fusion: Microstructure, Residual Stress and Property Gradients
}

\author{
S.C. Bodner, ${ }^{1, *}$ B. van de Vorst,${ }^{2}$ J. Zalesak,${ }^{3}$ J. Todt,${ }^{3}$ J.F. Keckes,${ }^{3}$ V. Maier-Kiener, ${ }^{4}$ \\ B. Sartory, ${ }^{5}$ N. Schell, ${ }^{6}$ J.W. Hooijmans, ${ }^{7}$ J.J. Saurwalt, ${ }^{7}$ and J. Keckes ${ }^{1}$ \\ ${ }^{1}$ Department of Materials Science - Chair of Materials Physics, \\ Montanuniversität Leoben, A-8700 Leoben, Austria \\ ${ }^{2}$ TNO, 5656 AE Eindhoven, the Netherlands \\ ${ }^{3}$ Erich Schmid Institute of Materials Science, Austrian Academy of Sciences, A-8700 Leoben, Austria \\ ${ }^{4}$ Department of Materials Science, Chair of Physical Metallurgy and Materials Testing, \\ Montanuniversität Leoben, A-8700 Leoben, Austria \\ ${ }^{5}$ Materials Center Leoben GmbH, A-8700 Leoben, Austria \\ ${ }^{6}$ Helmholtz Zentrum Geesthacht, Centre for Materials and Coastal Research, D-21502 Geesthacht, Germany \\ ${ }^{7}$ Admatec Europe BV, 5051 DV Goirle, the Netherlands
}

\begin{abstract}
Synthesis of multi-metal hybrid structures with narrow heat affected zones, limited residual stresses and secondary phase occurrence represents a serious scientific and technological challenge. In this work, liquid dispersed metal powder bed fusion was used to additively manufacture a multilayered structure based on alternating Inconel 625 alloy (IN625) and 316L stainless steel (316L) layers on a 316L base plate. Analytical scanning and transmission electron microscopies, high-energy synchrotron X-ray diffraction and nanoindentation analysis reveal sharp compositional, structural and microstructural boundaries between alternating $60 \mu \mathrm{m}$ thick alloys' sub-regions and unique microstructures at macro-, micro- and nano-scales. The periodic occurrence of IN625 and 316L subregions is correlated with a cross-sectional hardness increase and decrease and compressive stress decrease and increase, respectively. The laser scanning strategy induced a growth of elongated grains separated by zig-zag low-angle grain boundaries, which correlate with the occurrence of zigzag cracks propagating in the growth direction. A sharp $\langle 110\rangle$ fiber texture within the 316L regions turns gradually into a $\langle 100\rangle$ fiber texture in the IN625 regions. The occurrence of the C-like stress gradient with a pronounced surface tensile stress of about $500 \mathrm{MPa}$ is interpreted by the temperature gradient mechanism model. Chemical analysis indicates a formation of reinforcing spherical chromium-metal-oxide nano-dispersoids and demonstrates a possibility for reactive additive manufacturing and microstructural design at the nanoscale, as a remarkable attribute of the deposition process. Finally, the study shows that the novel approach represents an effective tool to combine dissimilar metallic alloys into unique bionic hierarchical microstructures with possible synergetic properties.
\end{abstract}

Keywords: Powder Bed Fusion; Microstructure and Stress; Multi-Metal Material; Stainless Steel; Inconel

\section{INTRODUCTION}

Multi-material hybrid structures in nature and technology combine properties of their individual constituents throughout complex microstructures to achieve synergistic effects, like improved mechanical properties and oxidation resistance, which go beyond the properties of their monophasic counterparts [1-3]. Typically, multilayered, composite and hierarchical microstructures are used to achieve improved functionality by combining materials with different physical properties [2, 4-7]. Similarly, functionally graded materials (FGMs) are designed to comply with spatially variable functional requirements like strength, oxidation resistance or toughness by adopting gradients of dissimilar phases, microstructure and/or residual stresses [8-10]. However, joining of metals into multi-metal hybrid structures with diffuse or abrupt in-

* sabine.bodner@unileoben.ac.at terfaces between the individual phases or alloys and narrow heat affected zones resulting in limited the first-order residual stress, macroscopic distortion and secondary phase formation represents still a serious technological challenge [11].

By their principle of a sequential layer-by-layer deposition, additive manufacturing technologies (AM) are ideally suited to manufacture parts with complex external and internal geometries [3, 12-14]. Additionally, powder and wire feed material supplies have been known to allow for an adjustment of volume fraction of metallic components [15-17]. In particular, the directed energy deposition (DED) approach, in which powder is fed into the melt-zone and molten at every layer by a laser, can be used to fabricate components with a variable layerby-layer composition and unique microstructures [18]. Within this contribution, however, a novel laser powder bed fusion-related technology, based on liquid dispersed metal powder, is used to produce a multi-metal hybrid structure. 
The fabrication of FGMs using AM technologies allows for generating property-specific part areas by matching the process parameters to the localized functionality [19]. It is however common that combining dissimilar metals using AM technology may result in the formation of compositional and microstructural inhomogeneities as well as in secondary phases and stress concentrations, which may decisively deteriorate part's functional properties [20-22].

A significant effort has been devoted to the development of multi-material additive manufacturing (MMAM) of hybrid structures, reviewed recently in Ref. [3]. Exemplary AM systems equipped with several powder feeders were used to prepare graded Ti-V [23, 24], TiMo [24], V-Ti6Al4V alloy [25], stainless steel-Ti6Al4V alloy [26] and Ti-6Al-4V/Ti-6.5Al-3.5Mo-1.5Zr-0.3Si [27] materials with novel refined microstructural features, aiming at aircraft and aero-engine applications. Similarly, bimetallic structures like Inconel 718-copper alloy GRCop-84 [28] and stainless steel 304L-Invar 36 [25] as well as gradient structures like $\mathrm{Ti}^{-\mathrm{TiO}_{2}}$ [29] and yttriastabilized zirconia-steel [30] were fabricated.

In the field of nuclear and steam power plants, aerospace and/or repair applications, especially high strength, corrosion and oxidation resistance and/or creep and fatigue resistance are required [31, 32]. For this reason, MM-AM of stainless steel (SS) and Inconel have been extensively explored [33, 34]. Carroll et al. [35] reported on fabrication of Inconel-steel FGM with diffuse compositional, structural and microstructural boundaries by applying gradually varying mixtures of Inconel 625 and grade 304L SS powders. The observed formation of cracks with a length of several hundred microns in a region with $79 \mathrm{wt} \% \mathrm{SS} 304 \mathrm{~L}$ and $21 \mathrm{wt} \%$ IN625 was attributed to the presence of secondary phases of transition metal carbides in the form of (Mo,Nb)C. Similarly, aiming at nuclear fission reactor applications, Hinojos et al. [36] studied the joinability of Inconel 718 and $316 \mathrm{~L}$ SS and vice versa utilizing electron beam melting (EBM) and reported abrupt compositional, structural and microstructural boundaries as well as low concentration of typical welding features. Cracking observed at the 316L/IN718 interface was attributed to the deformation constrains imposed by IN718. Additionally, using finite-element numerical analysis, Hofmann et al. [9] showed that a gradient transition from SS 304L to IN625 across an automobile valve stem are expected to exhibit ten times lower stress at the transitioning zone at $1000 \mathrm{~K}$ compared to a friction stir-welded joint of the same materials.

In general, all previous studies on FGMs combining nickel-based alloys and steel indicated complex processmicrostructure-stress-properties relationships [35, 36]. Additionally, FGMs produced with diffuse and abrupt interfaces comprise usually unique microstructures with secondary phases and precipitates like $(\mathrm{Mo}, \mathrm{Nb}) \mathrm{C}$, $\mathrm{M}_{23} \mathrm{C}_{6}, \mathrm{NbC}[35,36]$ etc. and there were cracks with lengths up to several $100 \mu \mathrm{m}$ observed systematically in the transition regions [35]. These findings indicate that there is a significant potential to further optimize the functional properties of the particular nickelbased alloys-steel FGMs by knowledge-based microstructural design, including a development of novel deposition routes and recipes.

For this work, the non-precipitation hardening alloy Inconel 625 (IN625) and stainless stee grade 316L (316L) are selected to fabricate a multi-metal hybrid structure. A relatively novel deposition technique based on liquid dispersed metal powder bed fusion is used to form a complex microstructure with multilayered morphology, zigzag grain boundaries, multiscale interfaces and ceramic nanoparticle reinforcement. The three-fold work objectives encompass (i) exploring the possibilities and advantages of the liquid powder bed fusion technology, (ii) analyzing gradients of phases, microstructure, composition and mechanical properties at multiple length-scales using correlative cross-sectional micro-analytics and (iii) additive manufacturing of multi-metal hybrid materials. Additionally, reactive additive manufacturing as an integral part of the liquid dispersed metal powder bed fusion process is introduced in order to indicate the possibility to tailor mechanical properties of the grown structures by integrating homogeneously dispersed nanoparticles.

\section{MATERIALS AND METHODS}

For the synthesis of a multi-layered IN625-316L structure on a $3 \mathrm{~mm}$ thick $316 \mathrm{~L}$ stainless steel base plate, a prototype system LASERFLEX Conflux based on liquid dispersed metal powder bed fusion (Fig. 1) was used. As input material, AISI 316L (specified with $\sim[65,12,18,2] \%$ of $\mathrm{Fe}, \mathrm{Ni}, \mathrm{Cr}$ and Mo, respectively, as well as other balancing elements with less than $\sim 1 \%$ concentration) and IN625 (specified with $\sim[58,20,8,4] \%$ of $\mathrm{Ni}, \mathrm{Cr}, \mathrm{Mo}, \mathrm{Fe}$ and $\mathrm{Nb}$, respectively, as well as other balancing elements with less than $\sim 1 \%$ concentration) metal particles with $d_{50}$-diameters of $\sim 4.2$ and $\sim 3.4 \mu \mathrm{m}$, respectively, were applied. In order to produce two powder suspensions, the particles were dispersed in an aqueous solution enriched with an ethanol-based micropolymer glue. Individual IN625 and 316L layers were produced by (i) lowering the building platform by $\sim 30 \mu \mathrm{m}$, (ii) depositing the particular suspension, (iii) wiping the metal suspension across the building platform, (iv) waiting for a few seconds in order to dry and densify the liquid powder bed in the inert nitrogen atmosphere and (v) melting the dried metal powder in the nitrogen atmosphere, using a laser source (cf. Fig. 1). The laser system and its optics provides radiation with a wavelength of $1064 \mathrm{~nm}$ and a spot size of $\sim 12 \mu \mathrm{m}$. A laser power of $137 \mathrm{~W}$, a scan speed of $500 \mathrm{~mm} / \mathrm{s}$, a hatch distance of $90 \mu \mathrm{m}$ and a hatch rotation angle of 66 degrees between the layers were used. These process parameters were identical for both alloys. The temperature of the base plate was kept at $\sim 25^{\circ} \mathrm{C}$. After the laser melting the described procedure from (i) to (v) was repeated to 
produce the next layer. For further microscopy analysis, the structure's cross-section was mechanically polished using a standard colloidal silica polishing suspension by Struers with a particle size of $0.04 \mu \mathrm{m}$ (O-PS polishing suspension).

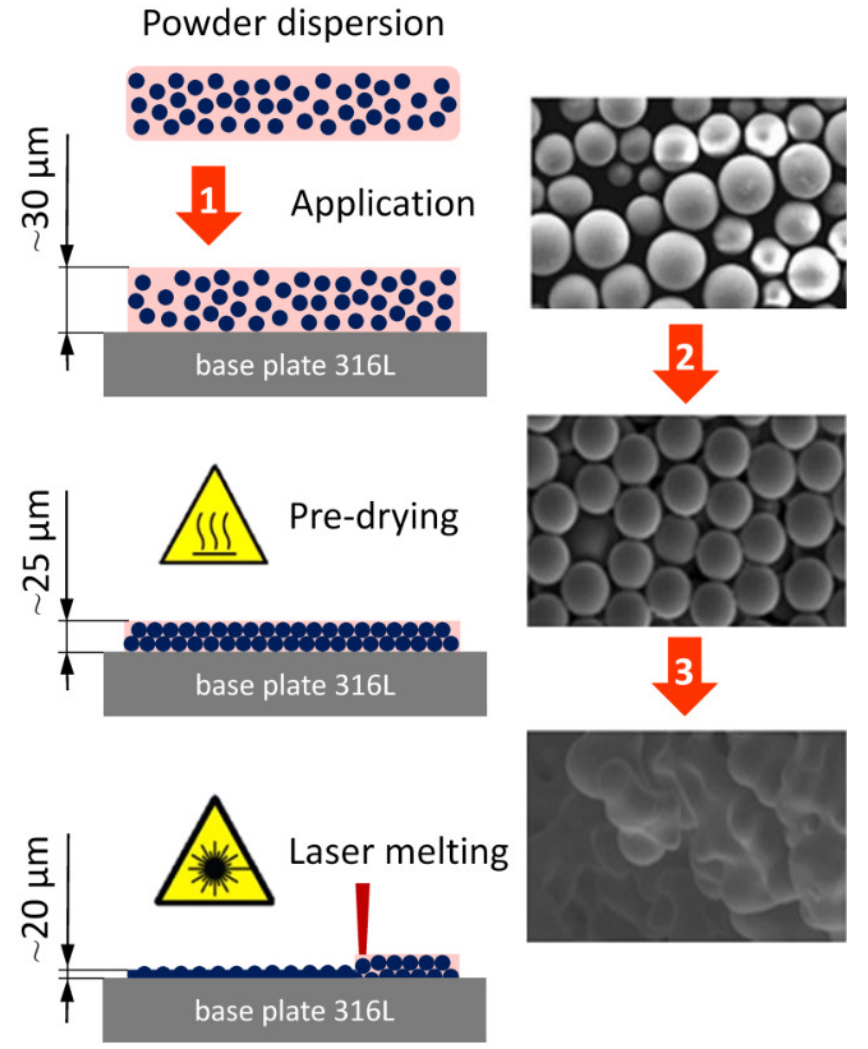

FIG. 1. A schematic description of the liquid dispersed metal powder bed fusion process. Within the first step, a suspension layer with a thickness of $\sim 30 \mu \mathrm{m}$ containing metallic $316 \mathrm{~L}$ or IN625 particles is applied on the 316L base plate or on already existing build-up. Then, the suspension dries in a nitrogen flow and densifies to a thickness of $\sim 25 \mu \mathrm{m}$. Finally, a laser source is used to fuse the particles together and to produce a new metallic layer of $\sim 20 \mu \mathrm{m}$ in thickness, which is reinforced with dispersed nanoceramic particles (cf. Sec. IIIF).

The approach from Fig. 1 was used to grow two multilayered IN625-316L plate structures, identical in their morphology, with overall dimensions of $\sim(15 \times 2 \times 7) \mathrm{mm}$ each, on a 316L SS base plate with dimensions of $\sim$ $(40 \times 40 \times 3) \mathrm{mm}($ Fig. 2a). Both structures possessed a complex irregular cross-sectional morphology introduced in Fig. 2b. The aim behind selecting this specific morphology was primarily to evaluate the accuracy of the deposition approach to fabricate relatively thin monoalloy layers, to obtain information on the intermixing of IN625 and 316L alloys, to analyze microstructural evolution at and near inter-alloy interfaces and to evaluate cross-sectional residual strain across the structure. Further on, experimental results obtained only from one structure will be presented.
Optical microscopy (OM) characterization was performed using an Olympus BX51 system. Scanning electron microscopy (SEM), electron backscatter diffraction (EBSD) and energy dispersive X-ray analysis (EDX) were performed using a Gemini SEM 450 and an AURIGA CrossBeam systems from Carl Zeiss. A cross-sectional transmission electron microscopy (TEM) lamella with a thickness of $\sim 50 \mathrm{~nm}$ was fabricated using focused ion beam (FIB) machining within the Zeiss Auriga system by applying an acceleration voltage of $30 \mathrm{kV}$ and currents in the range from $20 \mathrm{nA}$ to $50 \mathrm{pA}$. The lamella was extracted from a sample region containing an interface between the two alloys. Microstructural and chemical characterization of the lamella was performed using scanning TEM (STEM) mode in a probecorrected FEI Titan Themis platform operated at $200 \mathrm{kV}$ and equipped with a Gatan Enfinium ER spectrometer as well as FEI Super-X EDX four quadrant detectors. The collected EDX signal was treated using Bruker Esprit software applying built-in standards.

Nanoindentation experiments were conducted using a platform Nanoindenter G200 with a Berkovich diamond tip in strain-rate controlled mode. A sinusoidal load signal was superimposed during the continuous sample loading in order to record the contact stiffness. Tip shape and frame stiffness calibrations were performed on a regular base according the Oliver and Pharr method [37]. Arrays of indents with an indentation depth of $\sim 1 \mu \mathrm{m}$ and a minimum distance of $\sim 20 \mu \mathrm{m}$ between the indents were positioned over the R1-R4 regions. Hardness and indentation moduli were evaluated for the four regions as well as for bulk IN625 and 316L.

Synchrotron characterization of the multi-layered sample was performed at the high energy materials science (HEMS) beamline P07B of PETRA III at DESY in Hamburg, which is operated by Helmholtz Zentrum Geesthacht. The experimental setup is presented schematically in supplementary data. The sample characterization was performed using a monochromatic beam of $87.1 \mathrm{keV}$ photon energy and a cross-section of $\sim$ $20 \times 500 \mu \mathrm{m}$, which was directed approximately parallel to the interfaces between IN625 and 316L. To perform a scanning cross-sectional X-ray micro-diffraction experiment (CSmicroXRD), the sample was moved along the growth direction with an increment of $20 \mu \mathrm{m}$. Almost 400 diffraction patterns, each with multiple Debye-Scherrer (DS) rings, were recorded from the sample's cross-section in transmission geometry using a Perkin Elmer twodimensional (2D) flat panel detector of $2048 \times 2048$ pixels with a pixel pitch of $200 \mu \mathrm{m}$. The sample-detector distance was set to $\sim 1.3 \mathrm{~m}$. A dark current correction was applied to each exposure. The exact geometry and distance of the detector with respect to the sample's position was determined using a LaB6 calibration standard powder. Diffraction data were treated using the pyFAI software package developed at the European Synchrotron Radiation Facility [38] and evaluated using custom scripts written in Python. 

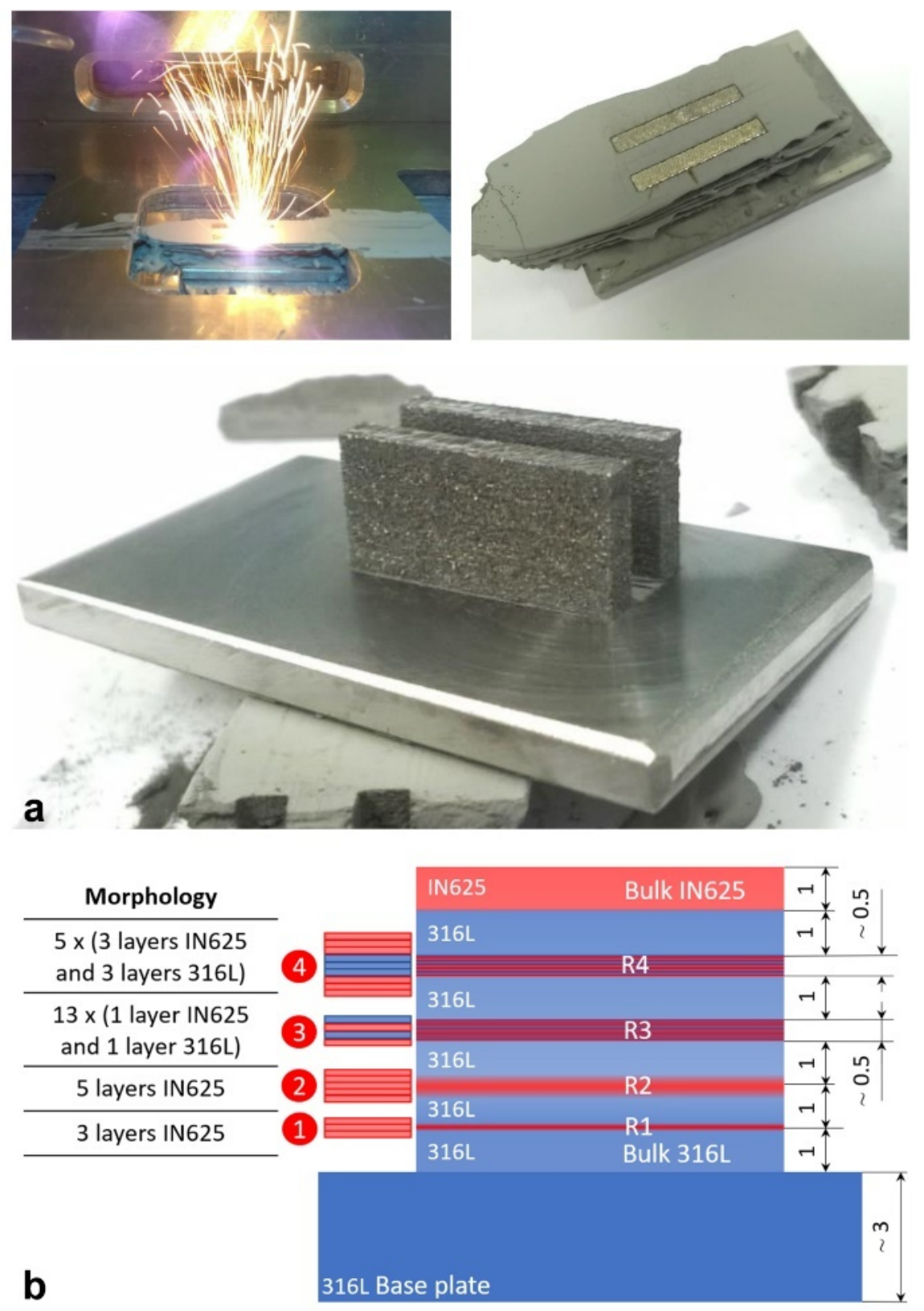

FIG. 2. Synthesis and geometry of the IN625-316L structure. (a) Various stages of the fabrication process to produce two identical multilayered plates. (b) On a 316L base plate, $\sim 1 \mathrm{~mm}$ thick layer of $316 \mathrm{~L}$ was grown followed by four regions R1-R4 including IN625, which are always separated by $\sim 1 \mathrm{~mm}$ of 316L. The deposition was finalized by $\sim 1 \mathrm{~mm}$ of IN625 on top. As indicated in (b) left, the R1-R4 regions consist of 3 (R1) and 5 (R2) layers of IN625, alternating IN625 and 316L layers in R3 and alternating groups of three IN625 and three 316L layers (R4). The thickness of every individual layer was set to $\sim 20 \mu \mathrm{m}$ (cf. Fig. 1).

\section{RESULTS}

\section{A. Cross-sectional morphology}

The morphology of the polished IN625-316L structure cross-section was analysed using OM and SEM. The OM micrograph in Fig. 3 shows the base plate, the five $1 \mathrm{~mm}$ thick regions of $316 \mathrm{~L}$, the regions $\mathrm{R} 1-\mathrm{R} 4$ and a $\sim 1 \mathrm{~mm}$ thick IN625 region on top (cf. also Fig. 2b). The interfaces between the layers appear to propagate parallel to the base plate surface and indicate that the microstructure is laterally homogeneous. The interface between the multi-layered structure and the base plate does not show any features of intermixing or dilution.

A variety of cracks were found at the structure's crosssection with a very typical zig-zag morphology. A rep- 
resentative example of one zig-zag crack is shown in the inset of Fig. 3 (cf. also OM and SEM micrographs in supplementary data). Comparable zig-zag cracks were found in the regions $\mathrm{R} 1-\mathrm{R} 4$ as well as in the top-most $\sim 1 \mathrm{~mm}$ thick IN625 region. Their occurrence can be correlated with the presence of IN625. The cracks propagated across the layers of both alloys within regions R1-R4 and approximately in the structure's growth direction, their origins will be discussed in Secs. III B and III C.

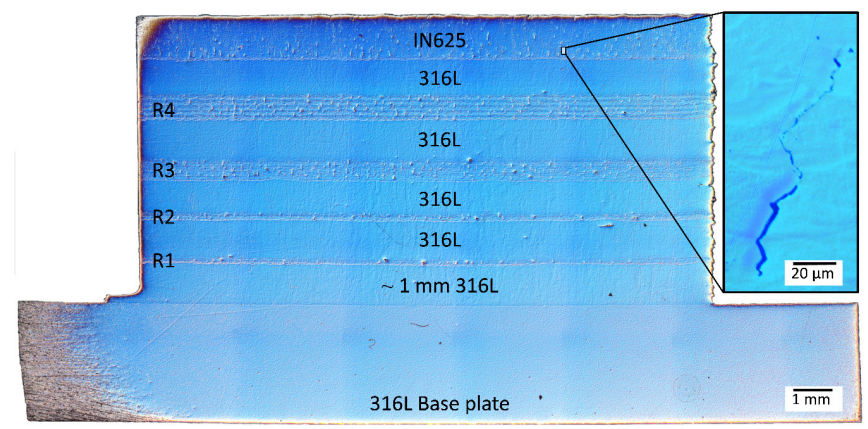

FIG. 3. OM micrograph showing the multi-layered IN625316L structure cross-section with $\sim 3 \mathrm{~mm}$ base plate, five $\sim 1 \mathrm{~mm}$ thick $316 \mathrm{~L}$ regions separated by the regions $\mathrm{R} 1-$ R4 with IN625 layers and layer-groups, respectively, and an IN625 region on top. The base plate's surface exhibits a concave bending. The inset at the right shows the cross-sectional morphology of a typical crack (cf. supplementary data).

The OM micrograph in Fig. 3 shows that the upper surface of the base plate shows a slight concave bending, which indicates the presence of compressive and tensile residual stress states within the top and bottom regions of the plate, respectively [39]. Consequently, it can be expected that the near-base plate region of the structure possesses tensile stresses which change gradually into compressive at the structure's surface. The stress formation is a result of the structure fabrication and its origins will be discussed in the next section. Fig. 3 as well as all SEM and TEM micrographs presented along the text are shown in full resolution in supplementary material.

\section{B. CSmicroXRD analysis}

In order to obtain grain-averaged information on the cross-sectional distribution of crystalline phases, texture and strain across the multi-layered structure, CSmicroXRD was carried out and volume-averaged data from the $2 \mathrm{~mm}$ thick sample (Fig. 2a) (along the X-ray beam direction) were collected. XRD data obtained by an azimuthal integration of the complete Debye-Scherrer rings in Figs. 4a and b show a cross-sectional evolution of IN625 and 316L reflections. Since both alloys possess face-centered cubic (fcc) lattice and lattice parameters of stress-relieved IN625 matrix and 316L steel differ only a few percent (depending on the actual alloy composition), fcc reflection positions (modified by the residual stress gradient) in Fig. 4a vary only marginally. Moreover, no other reflections from secondary intermetallic phases like $\gamma^{\prime}, \gamma^{\prime \prime}$ and $\delta$ in IN625 [40] or ferrite and carbide phases in 316L [41] can be identified in this phase plot. It should be however noted that quantitative XRD analysis is not sensitive to secondary phases with volume fractions smaller than 3-10\%, depending on the crystallite size. The evolution of IN625 and 316L 311-reflections in Fig. 4b reveals also the presence of the individual R1R4 layers, the top IN625 region as well as the base plate. The data in Fig. 4b allowed resolving also the presence of five groups of layers within region R4, which correlate with the multiple alternation of three IN625 and three 316L layers.

The layer-by-layer laser melting process, which is accompanied by the generation of localized high thermal loads during the metal powder bed fusion (Fig. 1), results in the formation of complex multiaxial residual stress distributions [42] whose tensile stress concentrations may significantly influence the mechanical stability of the fabricated parts. In the case of multi-metal hybrid structures like in Fig. 3, (i) thermal stresses are expected to be formed as a result of the mismatch of respective coefficients of thermal expansion (CTEs) of $\sim 15 \times 10^{-6} \mathrm{~K}^{-1}$ [43] and $\sim 18 \times 10^{-6} \mathrm{~K}^{-1}$ [44] at $100^{\circ} \mathrm{C}$ between IN625 and $316 \mathrm{~L}$ regions. Additionally, (ii) growth stresses are formed as a result of complex cross-sectional microstructure and stress evolution during the fabrication process accompanied by cyclic elasto-plastic deformation. As the presence of residual stress was indicated by the base plate's bending (Fig. 3), scanning CSmicroXRD was used to assess cross-sectional strain-stress distributions in the structure. The motivation was to obtain qualitative data and assess the role of particular microstructural features in the formation of stress. IN625 and 316L 311-Debye-Scherrer rings collected from the individual cross-sectional structure positions were evaluated with regard to their ellipticity. The obtained data were used to estimate depth gradients of X-ray elastic strains and residual stresses. For the evaluation, it was supposed that the shear X-ray elastic strains $\varepsilon_{i j}^{311}$ and shear residual stresses $\sigma_{i j}$ can be neglected for simplicity. In this case, the measured ellipticity is proportional to the difference of in-plane and out-of-plane residual stresses of $\sigma_{11}-\sigma_{33}$ and X-ray elastic strains $\varepsilon_{11}^{311}-\varepsilon_{33}^{311}$, which were evaluated using the Hill grain interaction model applying appropriate X-ray elastic constants $[45,46]$. Since the out-of-plane stresses $\sigma_{33}$ can be supposed to be relatively small, due to the free sample surface, the evaluated $\sigma_{11}-\sigma_{33}$ values from Fig. 4c can be therefore used to describe an evolution of the in-plane stresses $\sigma_{11}$ across the structure's cross-section.

The surface region of the base plate is in compression [39] and the stress profile across the multi-layered structure exhibits a complex oscillatory stresses behaviour, which is superimposed on a dominant C-shaped stress depth dependence (Fig. 4c). According to the temperature gradient mechanism (TGM) model [39, 42, 47], the 

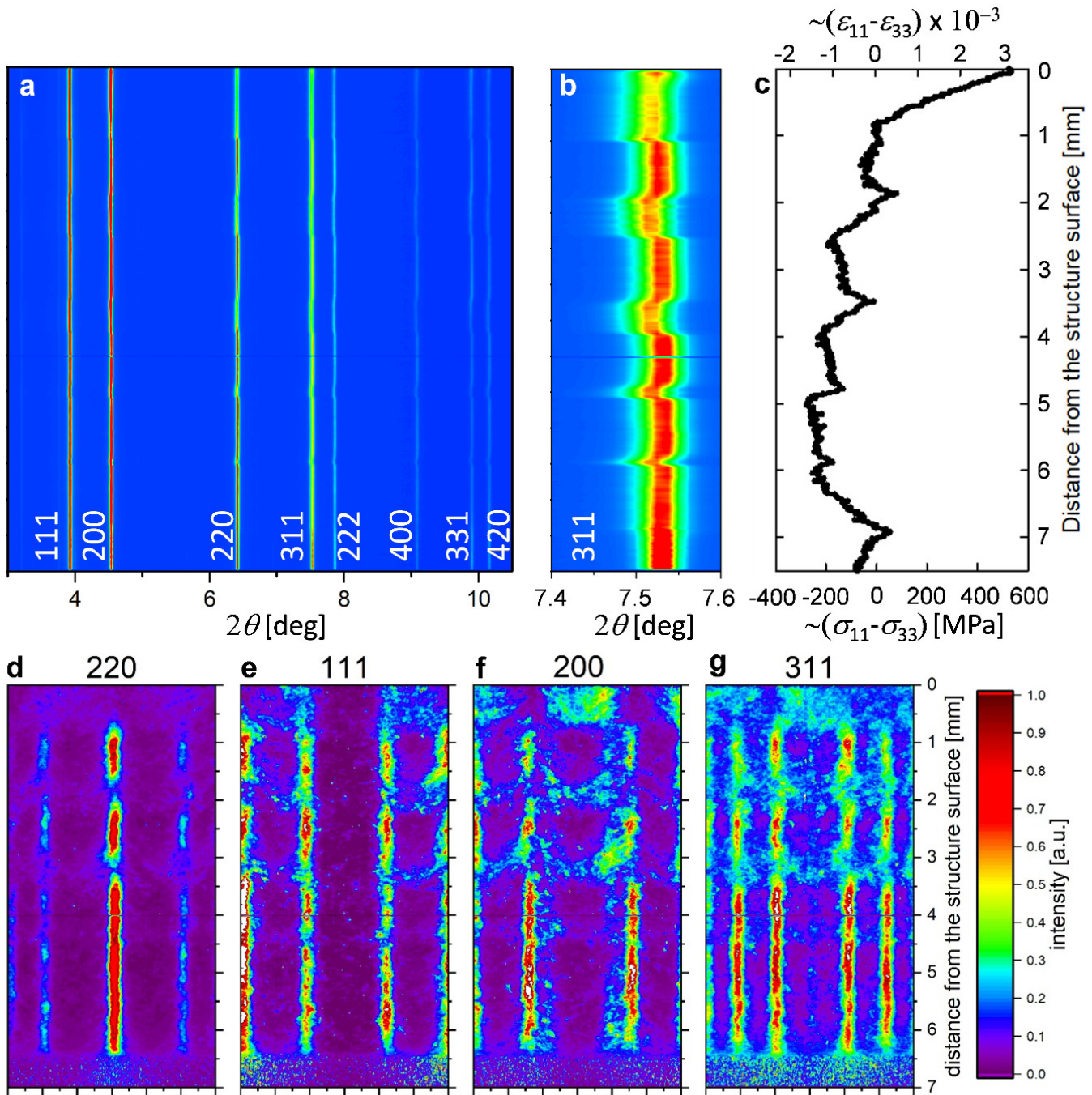

$\begin{array}{lllllllllllllllllllllllllllllllll}-90 & -60 & -30 & 0 & 30 & 60 & 90 & -90 & -60 & -30 & 0 & 30 & 60 & 90 & -90 & -60 & -30 & 0 & 30 & 60 & 90 & -90 & -60 & -30 & 0 & 30 & 60 & 90\end{array}$ azimuthal angle [deg] azimuthal angle [deg]

azimuthal angle [deg]

azimuthal angle [deg]

FIG. 4. Results from CSmicroXRD on the multi-layered structure. (a) The phase plot reveals only the presence of fcc reflections of IN625 and 316L alloys with very similar lattice parameters. (b) The evolution of IN625 and 316L 311 reflections shows the positions of R1-R4 regions, the top IN625 layer and the base plate. (c) The strain/stress plot indicates a dominant C-shaped stress depth dependence with compressive stress relaxations at the positions of R1-R4 regions. (d-g) The texture plots show the distributions of diffraction intensities along Debye-Scherrer rings for IN625 and 316L 220, 111, 200 and 311 reflections.

C-shaped stress profile across the multi-layered structure can be interpreted by the effect of the cyclic heating of the mechanically restrained preceding metallic layers, which results in the formation of compressive stresses and material plastification. During cooling down, tensile stresses are formed in the plastified surface zone (like in the topmost $\sim 1 \mathrm{~mm}$ thick IN625 region in Fig. $4 \mathrm{c}$ ), which are balanced by compressive stresses in the build-up zone beneath and in the base plate. Additionally, the oscillatory stress behaviour in Fig. 4c indicates that the growth of 316L layers results systematically in an increase of compressive stresses at all five cross-sectional positions in the structure. Conversely, the deposition of IN625 results in a systematic relaxation of compressive stresses. The re- sulting compressive stress oscillations can be interpreted by a higher degree of plastic strain generation in 316L compared to IN625, which is due to a lower yield strength of $316 \mathrm{~L}$, as well as by a stress relaxation in IN625 manifested by the formation of zig-zag cracks, as visible in Fig. 3. It is clear, however, that this qualitative explanation of the stress gradient formation is very simplified and the formation depends on a variety of process and material parameters like heat source path and heat transfer to the underlying layers, temperature distributions, local thermal conductivity, local yield strength and local CTEs. Moreover, due to their unique microstructures, materials' parameters are temperature- and, thus, laser power-dependent [17]. 
The plots in Figs. 4d-g show azimuthal distributions of diffraction intensities along IN625 and 316L 220, 200, 111 and 311 Debye-Scherer rings and document thus a qualitative evolution of crystallographic texture across the structure. Since the angle 0 and 90 degrees in Figs. $4 d-g$ correspond to the out-of-plane and in-plane orientations of the diffraction vectors, respectively, the results indicate the presence of $\langle 110\rangle$ fibre texture, which evolves gradually in the first 316L layer and saturates within $\sim 0.4 \mathrm{~mm}$ above the base plate. Thereafter, it is preserved in all subsequent $316 \mathrm{~L}$ layers (Fig. 3d). This is documented for instance by the strong azimuthal maxima of 220, 200 and 111 reflections at $\sim 0^{\circ}, \sim 45^{\circ}$ and $\sim 35^{\circ}$, respectively. These maxima correspond to the angles of $\sim 45^{\circ}$ and $\sim 35^{\circ}$ between (110) and (100) as well as (110) and (111) crystallographic planes, respectively. The occurrence of azimuthal maxima broadening at the positions of $\mathrm{R} 2, \mathrm{R} 3$ and $\mathrm{R} 4$ regions indicates that the addition of IN625 weakens the $\langle 110\rangle$ fibre texture, which practically disappears or even turns into a $\langle 100\rangle$ fibre texture within the top IN625 sublayer. The results also reveal that the base plate is not free from texture, but there is a certain type of a weak biaxial texture. This is represented in Figs. 4d, e by the occurrence of weak maxima at $\sim 0$ and $\sim 90$ degrees for 220 and 111 reflections, respectively.

\section{Local texture analysis}

In Fig. 5, EBSD micrographs show the cross-sectional evolution of crystallite orientations and microtexture along the in-plane and out-of-plane sample axes [100] and [001], respectively, at the interface between the base plate and the first 316L layer (Fig. 5e, f) and across the regions R1 (Fig. 5c, d) as well as R4 (Fig. 5a, b). The data in Fig. 5e document that the apparently homogenous microstructure of the base plate with globular grains with an average size of $\sim 12 \mu \mathrm{m}$ in diameter rapidly turns into a textured microstructure with elongated grains and an average grain length of $\sim 108 \mu \mathrm{m}$ within the first $316 \mathrm{~L}$ layers. In case of all five $\sim 1 \mathrm{~mm}$ thick $316 \mathrm{~L}$ layers, the EBSD data indicate the presence of a relatively pronounced $\langle 110\rangle$ fibre texture with random in-plane crystallite orientations (e.g. along [100] and sample direction), in agreement with the data from Figs. $4 \mathrm{~d}-\mathrm{g}$.

The region R1 (Figs. 5c, d) shows a very localized grain refinement and a small change in the crystallite orientation, which however seemingly does not influence the cross-sectional microstructure significantly. In other words, the insertion of the three IN625 layers (cf. Figs. 2b, 3) did not influence the epitaxial overgrowth of the individual layers resulting in the preservation of the columnar grain microstructure as well as texture. This is in agreement with the XRD results from Figs. $4 \mathrm{~d}-\mathrm{g}$, where also no significant texture disruption was observed within the region $\mathrm{R} 1$. The behaviour of the preserved crystallite microtexture can be interpreted by very simi- lar lattice parameters of both alloys, differing only a few percent, which clearly promoted a heteroepitaxial overgrowth.

The EBSD data from the region $\mathrm{R} 4$, however, indicate changes in the crystallite orientation (Figs. 5a, b) caused by the addition of IN625 sublayers, namely the $\langle 110\rangle$ fibre texture was gradually disrupted as can be seen especially in Fig. 5b. This observation is in agreement with the XRD data from Figs. 3d-g, which also showed that the deposition of multiple IN625 layers induces a gradual texture randomization.

Moreover, the EBSD results from Fig. 5 document a zig-zag cross-sectional morphology of elongated grains (and low-angle grain boundaries with a misorientation angle less than about 15 degrees), which is obtained as a result of the applied scanning strategy. Grain-boundary maps are also presented in the supplementary material. This zig-zag morphology can be correlated to the zig-zag cracks patterns observed using SEM and OM especially at the cross-sectional positions of the regions R1-R4 (cf. inset in Fig. 3). The zig-zag cracks are expected to originate from the particular shape of the grain boundaries and/or in-plane residual stresses induced by CTEs mismatch between the sublayers.

\section{Chemical analysis}

SEM and EDX analyses were performed in order to characterize microstructure and chemistry of both alloys within the individual layers. The results indicate a homogenous distribution of elements in both phases and, additionally, no microscopic precipitation resulting in a localized elemental enrichment or depletion, in agreement with the CSmicroXRD data (Fig. 4). Moreover, no formation of microscopic dendritic regions was observed at the microscale. This is in contrast to the results from similar studies on the IN718-304L systems, where the formation of microscopic enrichments in $\mathrm{Nb}$ and $\mathrm{Mo}$, with a few shared areas containing $\mathrm{C}$, was observed inter- and intragranularly in IN718 and IN625 [35, 36]. This discrepancy can be interpreted by the different thermal treatments applied in Refs. [35, 36] and in the present process.

EDX concentration mapping of the region $\mathrm{R} 4$ was performed to examine the intermixing of the elements between IN625 and 316L sub-regions during the multilayer structure fabrication. In Fig. 6, distributions of major elements indicate the presence of ten sub-regions, each consisting of three layers of IN625 or 316L. Both deposited alloys contain $\mathrm{Fe}, \mathrm{Ni}, \mathrm{Cr}$ and Mo elements, whereas $\mathrm{Nb}$ is initially present only in IN625. In the Nb concentration map in Fig. 6, it is possible to resolve five $\mathrm{Nb}$-rich sub-regions representing IN625 and four 316L sub-regions with a smaller amount of $\mathrm{Nb}$. Moreover, there is evidence of a higher concentration of $\mathrm{Nb}$ in the $\sim 1 \mathrm{~mm}$ thick $316 \mathrm{~L}$ region above region $\mathrm{R} 4$, compared to the $\mathrm{Nb}$ (EDX background) concentration level in the $\sim 1 \mathrm{~mm}$ thick $316 \mathrm{~L}$ region, below region $\mathrm{R} 4$. These observations indicate 

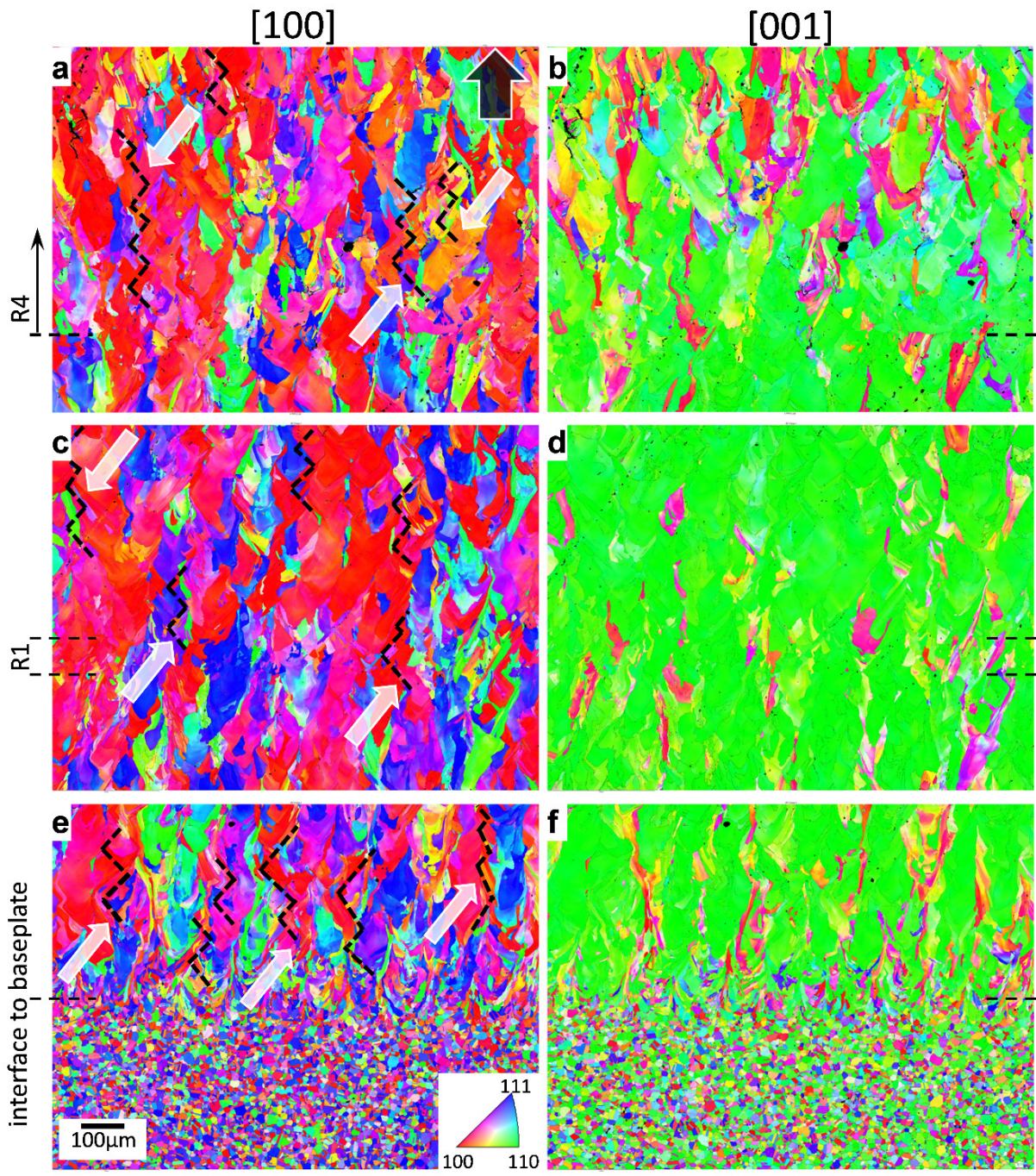

FIG. 5. EBSD data show the orientation of the crystallites with respect to the in-plane and out-of-plane sample axes [100] and [001], respectively, at the interface between the base plate and the first 316L layer (e, f) and across the regions R1 (c, d) as well as R4 $(\mathrm{a}, \mathrm{b})$. These microtexture data indicate the presence of a columnar grain morphology and a $\langle 110\rangle$ fibre texture in both alloys (cf. also supplementary data). The zig-zag patterns in the microstructure are indicated by black, dotted lines and white arrows (a, c, d). The dark arrow in (a) indicates the growth direction.

that after the deposition of IN625 layers, the elements of this alloy were distributed along the growth direction upwards as a result of out-diffusion into the melt-pool. Probably, the concentration of the IN625 alloying elements decreases exponentially with distance from IN625 layers.

The $\mathrm{Ni}$ and Fe concentration maps in Fig. 6 indicate very clearly the presence of five IN625 and five 316L layer-groups in region R4. In both types of sub-regions a simultaneous occurrence of $\mathrm{Fe}$ and $\mathrm{Ni}$ was evaluated. This effect can be attributed to an intermixing of the two elements between the deposited sub-regions and to the (intrinsic) simultaneous occurrence of the elements in both alloys. The following effects are remarkable however: The top-most sub-region of three layers of $316 \mathrm{~L}$ in region R4 (cf. Fig. 2b) shows lower and higher con- centrations of $\mathrm{Fe}$ and $\mathrm{Ni}$, respectively, compared to the neighboring top-most $\sim 1 \mathrm{~mm}$ thick $316 \mathrm{~L}$ region (Fig. 6 ). Similarly, also the bottom-most sub-region of three 316L layers in region R4 (following the first IN625 layer, cf. Fig. 2b) shows lower and higher concentrations of $\mathrm{Fe}$ and $\mathrm{Ni}$, respectively, compared to the underlying $\sim 1 \mathrm{~mm}$ thick 316L region, which appears to be almost free of $\mathrm{Ni}$ (Fig. 6). These observations also indicate that growth of the IN625 and 316L sub-regions was accompanied be cyclic remelting of the alloys and an intermixing of the elements between the layer being deposited and preceding layers.

Since IN625 contains an amount of Mo that is approximately two times higher than that in $316 \mathrm{~L}$ steel, Mo enrichments in the concentration map in Fig. 6 can be correlated with the occurrence of IN625 layers in region 

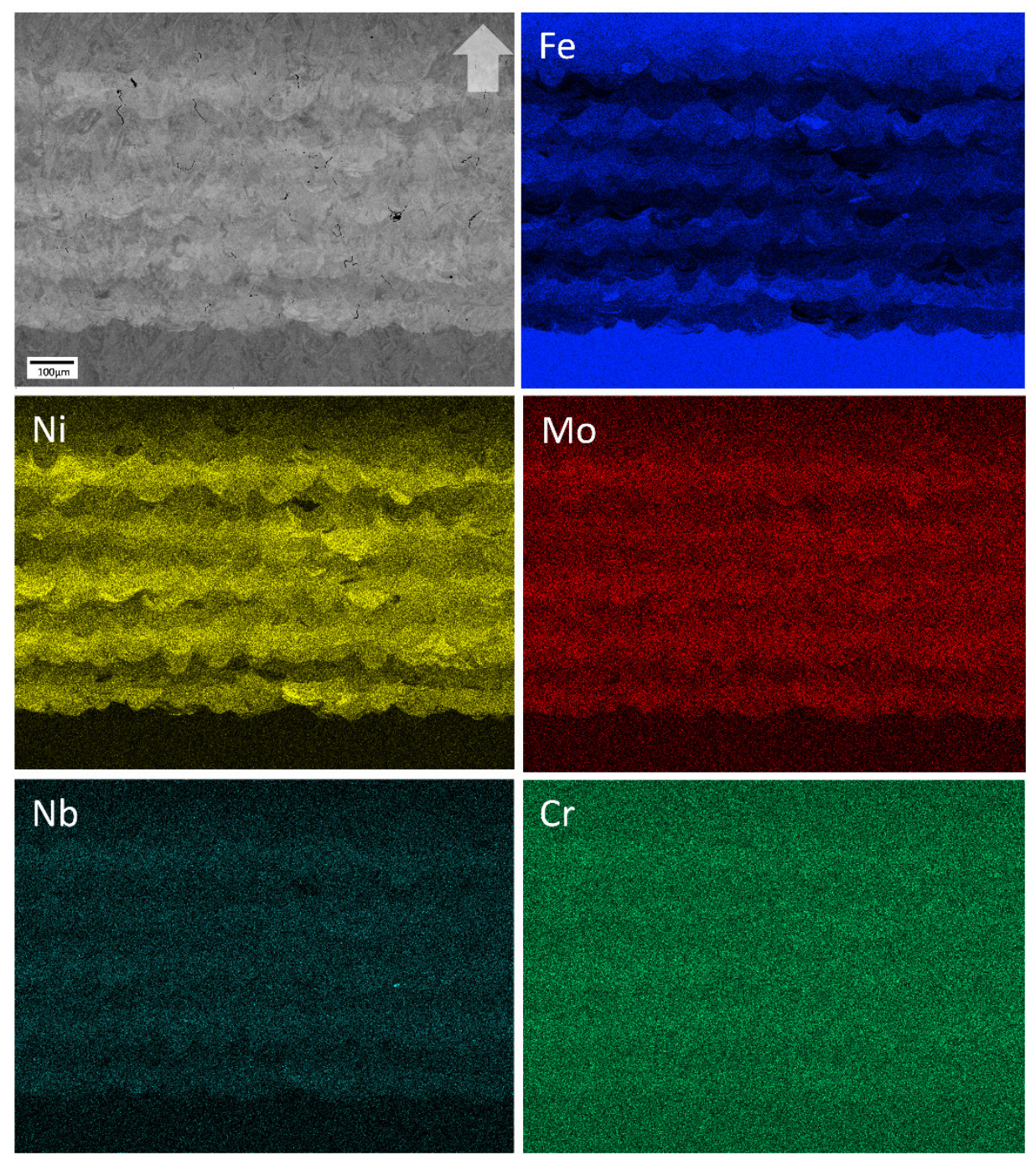

FIG. 6. EDX concentrations maps show the distribution of elements in the area of region R4. The arrow in the SEM micrograph indicates the building direction.

R4. The homogenous distribution of $\mathrm{Cr}$ across the region R4 (Fig. 6) correlates well with $\sim 20 \%$ amount of $\mathrm{Cr}$ in both alloys (cf. also Sec. III E).

An attempt was made to relate the occurrence of the cross-sectional cracks (Fig. 3) with the chemical gradients across the multilayered sample [35], but no such correlation was observed. In other words, a local fluctuation of the elemental concentrations was not found to be responsible for the zig-zag crack growth behavior, in contrast to the results of Carroll et al. [35], where microscopic Nband Mo- enrichment was observed within the cracked regions in IN625-304L system.

\section{E. Mechanical characterization}

Nanoindentation measurements were performed across the $\mathrm{R} 4$ region in order to evaluate the spatial distribution of hardness and reduced elastic modulus (the latter is presented in supplementary material). In Fig. 7, the cross-sectional distribution of hardness in the range of $\sim(3-4) \mathrm{GPa}$ is superimposed on an occurrence of Ni determined using EDX analysis (from Fig. 6). The data indicates that the multiple hardness increase can be correlated with the occurrence of $\mathrm{Ni}$ within the five groups of IN625 layers. In four sub-regions, each consisting of three 316L layers, as well as on the borders of region $\mathrm{R} 4$, the hardness decreases systematically. The regions R1-R3 showed the same behavior, where the insertion of IN625 interlayers caused an increase in hardness.

Hardness measurements were performed also on the sample (bulk) regions which consist exclusively of IN625 and $316 \mathrm{~L}$, where hardness values of $3.95 \pm 0.08$ and $2.81 \pm 0.08 \mathrm{GPa}$ were found, respectively, in relatively good agreement with the literature values of $\sim 4.3 \mathrm{GPa}$ [48] and $\sim 3.5 \mathrm{GPa}[49]$. This means that the bulk IN625 and 316L regions possess lower and higher hardness than the IN625 and 316L sub-regions in region R4, respec- 


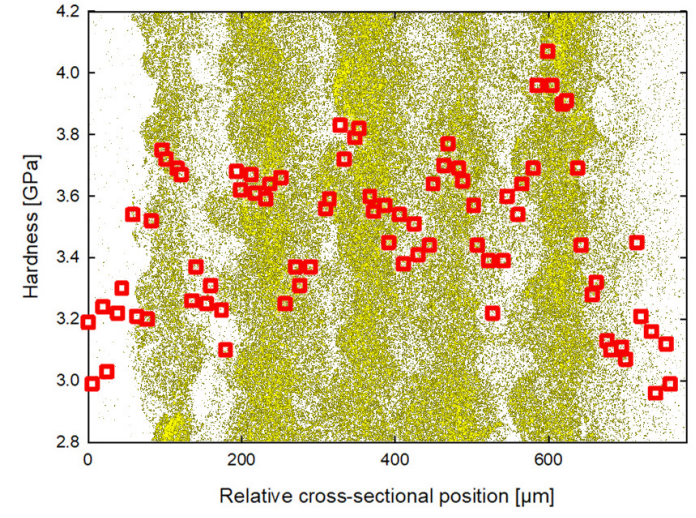

FIG. 7. Cross-sectional distribution of hardness across the region $\mathrm{R} 4$ superimposed on the elemental distribution of $\mathrm{Ni}$ from Fig. 6. The hardness increase correlates with the positions of five IN625 sub-regions.

tively. This finding can be interpreted by the elemental intermixing (cf. Fig. 6) and/or by different chemical compositions of $\mathrm{CrMO}_{x}$ dispersoids formed within region $\mathrm{R} 4$ (cf. Sec. IIIF).

\section{F. TEM characterization}

TEM characterization was performed in order to analyze the local microstructure and chemical composition across an exemplary interface, between the upper part of the $\sim 1 \mathrm{~mm}$ thick 316L region and the topmost IN625 region (Figs. 2b, 3). Bright field and high-angle annular dark-field (HAADF) micrographs revealed the presence of morphologically sharp interface between both alloys with a large amount of spherical nanodispersoids distributed throughout the interface region and an occurrence of heavy elements primarily at the IN625 grain boundaries, as documented by the HAADF micrograph in Fig. 8.

However, EDX analysis of the interface indicated gradual changes in the concentrations of $\mathrm{Fe}$ and $\mathrm{Ni}$ at a length scale of several $\sim 100 \mathrm{~nm}$ across the interface (Fig. 8). At the IN625 side, grain boundaries were revealed to contain enrichments of $\mathrm{Nb}, \mathrm{Mo}$ and $\mathrm{C}$ as result of nano-segregation, which is usually observed after thermal treatments of Inconel alloys [39]. At the 316L side, the segregation of $\mathrm{Nb}, \mathrm{Mo}$ and $\mathrm{C}$ at the grain boundaries was observed to a much smaller extent and $\mathrm{Nb}$ as well as $\mathrm{Si}$ enrichments within the dispersoids were detected (cf. Fig. 8).

Also a detailed EDX analysis of the spherical dispersoids with a diameter of $\sim 20-100 \mathrm{~nm}$ was performed (Fig. 9) at both sides of the interface. As documented by EDX maps in Fig. 9, the dispersoids were identified to be chromium-metal-oxide nanoparticles CrMOx enriched with $\mathrm{Si}$ and exhibiting Nb-rich shells. Generally, the dispersoids were found in both alloys across the whole structure at high concentrations and their actual composition depends on the chemistry of the particular alloy's matrix. This indicates that the dispersoid's formation is a characteristic attribute of the particular fusion process and resembles the formation of protective oxide scale rich in $\mathrm{Cr}$ in stainless steels with a relatively large fraction of $\mathrm{Cr}$ [50]. In other words, the liquid dispersed metal powder bed fusion induces an oxidation of $\mathrm{Cr}$ present in both alloys, resulting in the formation of dispersoids with some traces of $\mathrm{Si}$ and $\mathrm{Nb}$ (Fig. 9). This phenomenon will be denoted as reactive additive manufacturing. The ceramic dispersoids represent nanoscopic alloy reinforcements, which are expected to be favorable for the mechanical properties of the structure. Therefore reactive additive manufacturing as a part of the liquid dispersed metal powder bed fusion can be used as an effective tool to homogeneously incorporate nanoscopic particles across deposited structures in order to tailor mechanical properties.

\section{DISCUSSION}

This study demonstrates the feasibility of fabricating multi-layered IN625-316L structures (Figs. 2b, 3) with sharp structural and microstructural boundaries between the two alloys, deposited using liquid dispersed metal powder bed fusion (Fig. 1), where the alloys alternation was achieved at meso- and micro-scales (Figs. 3,6). The implementation of regions with periodically varying mechanical properties is expected to hinder crack propagation and may results in the synthesis of damage-resistant materials $[51,52]$. Due to the out-diffusion of alloying elements in the growth direction, however, an elemental intermixing was observed at length scales of several $100 \mathrm{~nm}$ (Fig. 8). The results from the multilayered region R4 (Figs. 3-6) document that the approach allows for the deposition of spatially alternating alloys with the resolution of $\sim 60 \mu \mathrm{m}$ in the growth direction, which consequently exhibits an oscillating hardness depth profile with a period of $\sim 100 \mu \mathrm{m}$ (Fig. 7).

The 316L layers possess a $\langle 110\rangle$ fiber texture, which saturates already in the first $\sim 1 \mathrm{~mm}$ thick $316 \mathrm{~L}$ region at a distance of $\sim 0.4 \mathrm{~mm}$ (Fig. 4e) above the base plate. However, the deposition of IN625 alloy induces a gradual disruption of the $\langle 110\rangle$ texture and the formation of a much weaker $\langle 100\rangle$ fiber texture like in Ref. [36], which is present especially in the top-most IN625 sublayer (Figs. $4 \mathrm{~d}-\mathrm{g}$ ). The deposition of IN625 induces moreover a refinement of the microstructure, which is visible already in region $\mathrm{R} 1$ (Figs. 5c, d).

The occurrence of a $\mathrm{C}$-shaped stress gradient with a negligible near-base plate stress, the oscillating compressive stresses at a level of $\sim-150 \mathrm{MPa}$ and the pronounced surface tensile stress of $\sim 500 \mathrm{MPa}$ (Fig. 3c) are interpreted by means of the TGM model [39, 42, 47], supposing cyclic material plastification and densification during the additive deposition. The $\mathrm{C}$-shaped stress profile 


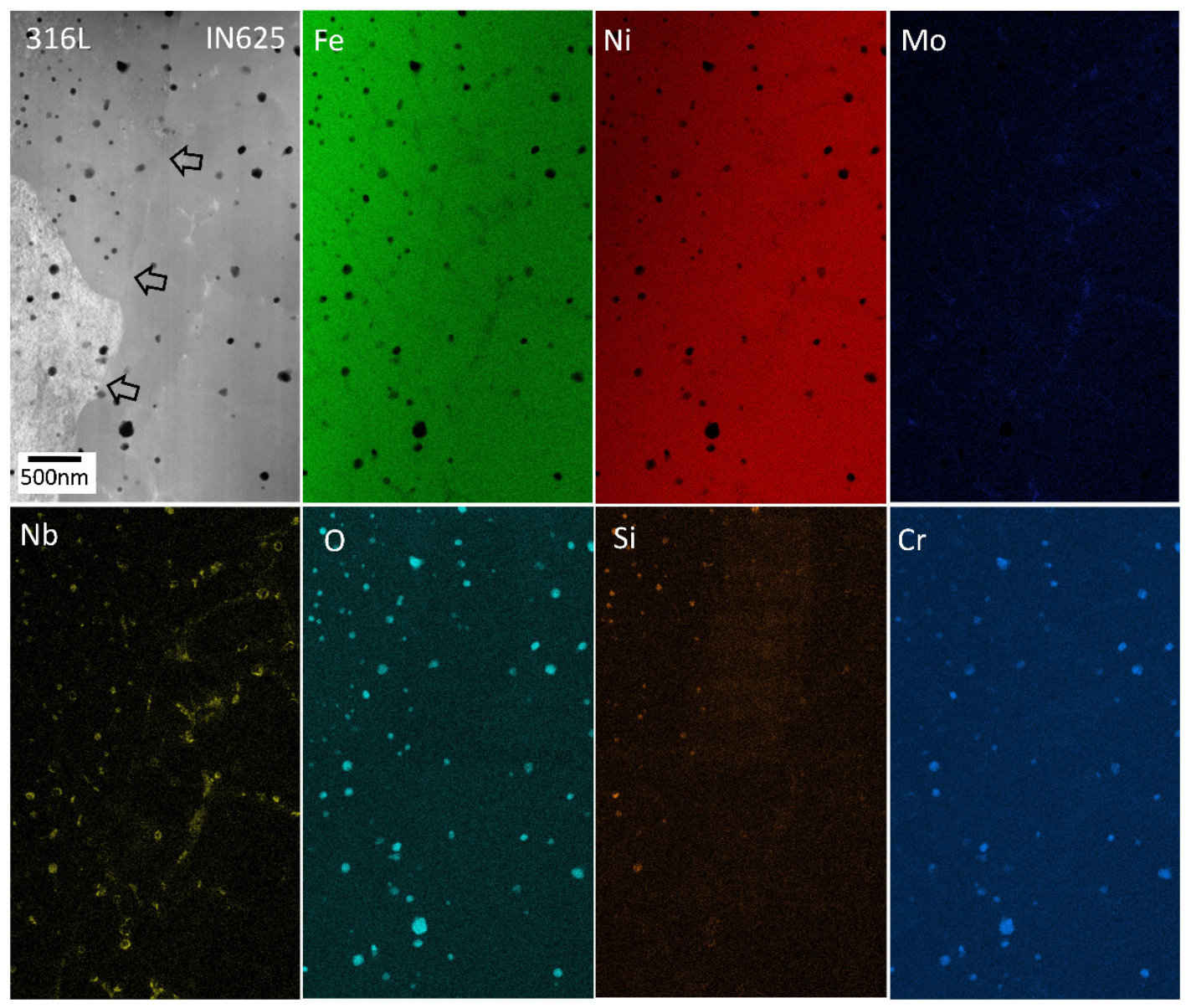

FIG. 8. EDX concentration maps show distributions of elements at the interface between 316L and IN625 alloys, which is indicated by arrows in the HAADF micrograph. Besides gradual changes in Fe and Ni concentrations and in the composition of $\mathrm{CrMO}_{x}$ dispersions, Nb-rich precipitates change their shape as a function of the Nb-content in the base material.

is a result of the mechanical equilibrium achieved after cooling down from the process temperature of compressively and tensilely stressed and mutually restrained surface and underlying bulk structure regions, respectively, whose stress signs are expected to almost reverse at room temperature. The deposition of IN625 layers results in the relaxation of compressive residual stresses (Fig. 3c), as a result of the supposed lower degree of plastic strain generation in IN625 and zig-zag cracking, which penetrates layers of both alloys (cf. inset in Fig. 3 and supplementary data) along zig-zag low-angle-grain boundaries. This effect is denoted as "ductility dip crackin" and appears as a result of IN625's ductility decrease during cooling down from the process temperature at straight or non-tortuous boundaries [36, 53].

EDX analysis in SEM (Fig. 6) indicated the absence of microscopic dendrites or precipitates with metallic enrichments/depletions zones in both alloys, in agreement with the CSmicroXRD data (Fig. 4a). Also no cracks were observed, which could be correlated with localized elemental enrichment or depletion. This is different from the results of Hinjos et al. [36] and Carroll et al. [35], where microscopic precipitates and features were reported in the same and/or similar alloys and which were correlated also with the occurrence of microscopic cracks. At the nanoscale, however, the EDX analysis performed in TEM indicated the presence of Nb, Mo and C enrichments caused by nano-segregation at IN625 grain boundaries (Fig. 8). These observations indicate that the liquid dispersed metal powder bed fusion process is favorable for the deposition of complex alloys because it is possible to suppress the formation of secondary phases at the microscale. Probably, due to the shorter diffusion lengths predefined by the particular laser design, the segregation of elements was limited and occurred only to a very small extent at the grain boundaries of IN625, as shown in Fig. 8.

IN625 regions and the top layer showed the presence of undesirable zig-zag cracks (Fig. 3), which may obviously negatively influence mechanical stability of the IN625SS316L build-up. Therefore a further optimization of the process is needed. The possibilities are to include additives into the powder suspension that will help to reduce solidification cracking. Another approach would be to increase the lasers pulse frequency, inspired by the results of Patterson et al. [54]. 

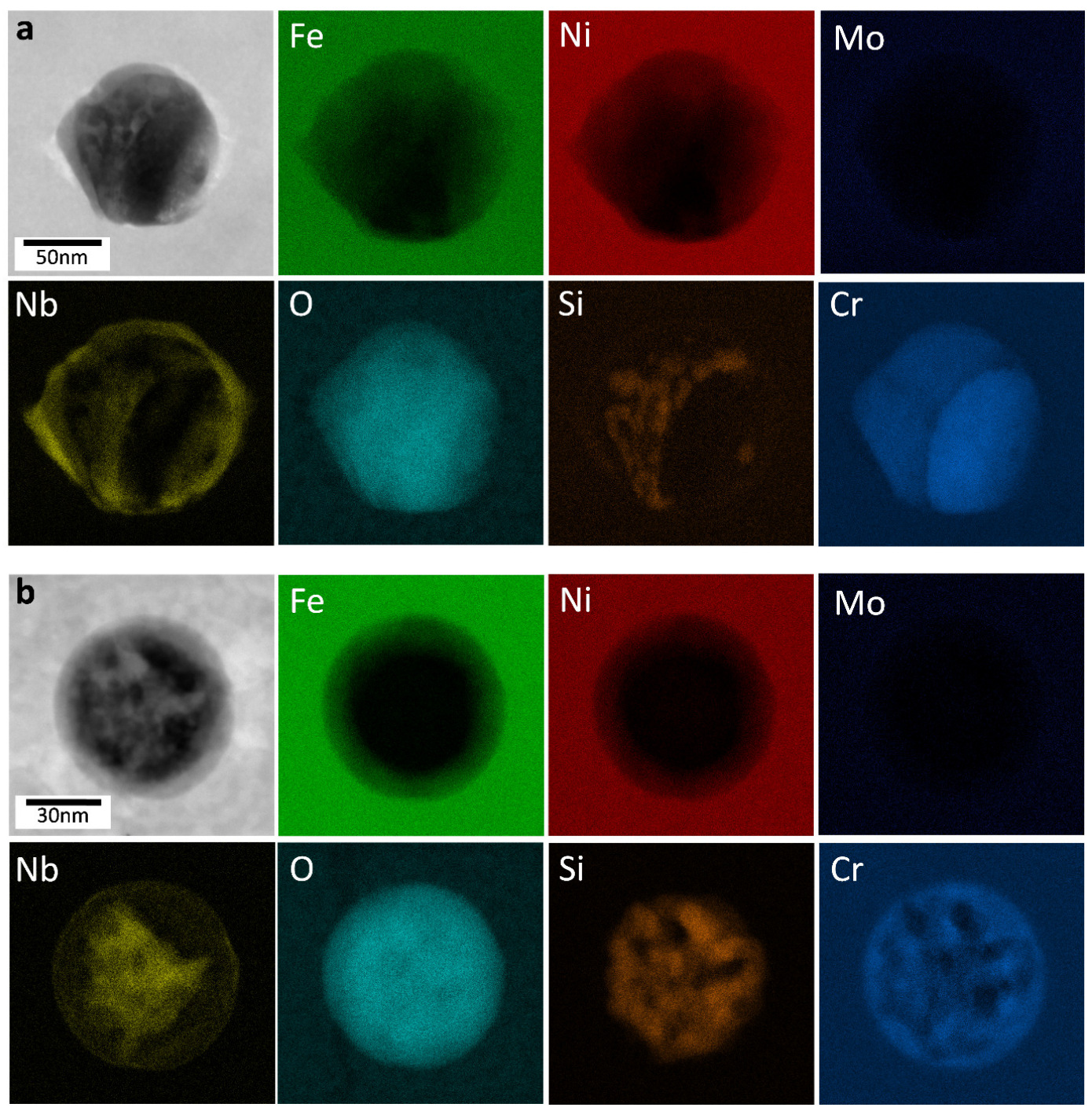

FIG. 9. EDX concentration maps show distributions of elements within representative dispersoids in IN625 (a) and 316L (b) alloys near the interface shown in Fig. 8. The spherical $\mathrm{CrMO}_{x}$ nanodispersoids possess Nb-rich shells and Si concentration appears to depend on the matrix alloy (cf. also Fig. 8).

A large concentration of spherical dispersoids with a diameter of $\sim 20-100 \mathrm{~nm}$, existing across the whole structure, was identified as a remarkable attribute of the liquid dispersed metal powder bed fusion process, which therefore includes also reactive additive manufacturing of ceramic nanodispersoids. The dispersoids could be found in both alloys and were identified mainly as $\mathrm{CrMO}_{x}$ precipitates, enriched with $\mathrm{Si}$ and $\mathrm{Nb}$, depending on the local alloy matrix chemistry. Their complex internal morphology is presented in Fig. 9. The dispersoid's formation is interpreted by the oxidation of $\mathrm{Cr}$ and other elements and resembles the formation of protective $\mathrm{Cr}_{2} \mathrm{O}_{3}$ oxide layers in Cr-rich stainless steels [50]. The dispersoids represent another hierarchical level within the alloy's microstructure and actually provide structural reinforcement. Their formation indicates another interesting feature of AM technologies, namely the possibility to perform reactive additive manufacturing and microstructural design at the nanoscale. In other words, the deliberate formation of oxide nanoparticles during the liquid dispersed metal powder bed fusion can be used as an effective tool to tune mechanical properties of AM-prepared alloys.

The multilayered structure (Fig. 3) exhibits a variety of bio-inspired attributes. The hatch strategy was selected to deposit microstructure with elongated grains and zigzag low-angle grain boundaries (Fig. 5). Zig-zagging interfaces between crystallites and microstructural features are very common in natural materials and serve to enhance fracture toughness while preserving strength, for instance in Saxidomus purpurata shells with zig-zag oriented aragonite platelets. The deposition of the alternating alloys at meso- (Fig. 3) and micro-scales (Fig. 6a) with different mechanical properties and oscillating stress gradients [51] at the cross-section (Fig. 3) resembles a 
nacre-like microstructure [55] and has been known to improve the mechanical properties of hybrid-materials. Even though the mismatch in mechanical properties of IN625 and 316L is not sufficiently high, this work shows the possibility to tailor multilayer materials using the liquid dispersed metal powder bed fusion approach. Additionally, the incorporation of ceramic nano-dispersoids (Figs. 8, 9) based on Cr oxides can be used as an effective tool to tune the mechanical properties of the AM structure by nanoscale design.

In summary, the liquid dispersed metal powder bed fusion approach offers the possibility to produce hierarchical microstructures based on meso- and micro-scale multilayers, grain boundaries of zig-zag shape, gradually changing textures, oscillatory stress concentrations and nanoscopic reinforcements, which together comprise a variety of multi-scale interfaces and can be used to tailor mechanical properties of AM structures.

\section{A. Conclusion}

This study demonstrates the feasibility of synthesizing a multi-metal hybrid structure based on IN625 and $316 \mathrm{~L}$, featuring unique multilayered microstructures at meso- and micro-scales, zig-zag shaped elongated grains and grain boundaries, regularly distributed nanoscopic dispersoids, complex texture evolution with gradual transitions, oscillating cross-sectional residual stresses and abrupt variation of mechanical properties across the multilayer. The zig-zag cracking correlated with the occurrence of IN625 (Fig. 2) as well as the pronounced Cshaped residual stress gradient (Fig. 3c), however, indicate the importance of process optimization in the fabrication of multi-metal hybrid materials with crack-free microstructures.

Although the synthesis of the multilayered structure (Fig. 3) was inspired by the architectural principles observed in biological materials [56] and the structure possesses a variety of interesting bionic microstructural fea- tures, the hardness values of IN625 and 316L do not differ significantly. Thus, effects like crack deflection toughening (observed in nacre) at the interfaces between IN625 and $316 \mathrm{~L}$ layers and regions could not be achieved here. The fabrication of hybrid systems consisting of materials with significantly different intrinsic mechanical properties like metal-ceramic composites has future potential to design multi-material structures with superior mechanical properties like high strength and toughness.

Finally, the presence of unique microstructures at meso-, micro- and nano-scales observed within the IN625316L multilayer (Fig. 2) indicates the necessity of using cutting edge analytical techniques operating at multiple length scale in order to reveal the very particular process-microstructure-stress-property relationships in AM structures.

\section{Acknowledgment}

A part of this work was supported by Österreichische Forschungsförderungsgesellschaft $\mathrm{mbH}$ (FFG), Project No. 861496, "CrossSurfaceMech". Financial support by the Austrian Federal Government (in particular from Bundesministerium für Verkehr, Innovation und Technologie and Bundesministerium für Wissenschaft, Forschung und Wirtschaft) represented by Österreichische Forschungsförderungsgesellschaft $\mathrm{mbH}$ and the Styrian and the Tyrolean Provincial Government, represented by Steirische Wirtschaftsförderungsgesellschaft mbH and Standortagentur Tirol, within the framework of the COMET Funding Programme is gratefully acknowledged. Part of the research leading to this result has been supported by the project CALIPSOplus under the Grant Agreement 730872 from the EU Framework Programme for Research and Innovation HORIZON 2020. Another part of this work was carried out with the support of CEITEC Nano Research Infrastructure (ID LM2015041, MEYS CR, 2016-2019), CEITEC Brno University of Technology.
[1] K. Martinsen, S. J. Hu, B. E. Carlson, Joining of dissimilar materials, CIRP Ann. - Manuf. Technol. 64 (2) (2015) 679-699.

[2] P. Fratzl, R. Weinkamer, Nature's hierarchical materials (nov 2007).

[3] A. Bandyopadhyay, B. Heer, Additive manufacturing of multi-material structures (jul 2018).

[4] W. J. Clegg, K. Kendall, N. M. N. Alford, T. W. Button, J. D. Birchall, A simple way to make tough ceramics, Nature 347 (6292) (1990) 455-457.

[5] J. W. Dunlop, P. Fratzl, Biological composites, Annu. Rev. Mater. Res. 40 (1) (2010) 1-24.

[6] R. Hahn, M. Bartosik, R. Soler, C. Kirchlechner, G. Dehm, P. H. Mayrhofer, Superlattice effect for enhanced fracture toughness of hard coatings, Scr. Mater. 124 (2016) 67-70.
[7] Y. M. Wang, T. Voisin, J. T. Mckeown, J. Ye, N. P. Calta, Z. Li, Z. Zeng, Y. Zhang, W. Chen, T. T. Roehling, R. T. Ott, M. K. Santala, P. J. Depond, M. J. Matthews, A. V. Hamza, T. Zhu, Additively manufactured hierarchical stainless steels with high strength and ductility, Nat. Mater. 17 (1) (2018) 63-70.

[8] A. Mortensen, S. Suresh, Functionally graded metals and metal-ceramic composites: Part 1 processing, Int. Mater. Rev. 40 (6) (1995) 239-265.

[9] D. C. Hofmann, J. Kolodziejska, S. Roberts, R. Otis, R. P. Dillon, J. O. Suh, Z. K. Liu, J. P. Borgonia, Compositionally graded metals: A new frontier of additive manufacturing, J. Mater. Res. 29 (17) (2014) 1899-1910.

[10] Y. Liu, F. Weng, G. Bi, Y. Chew, S. Liu, G. Ma, S. K. Moon, Characterization of wear properties of the functionally graded material deposited on cast iron by laser- 
aided additive manufacturing, Int. J. Adv. Manuf. Technol. 105 (10) (2019) 4097-4105.

[11] Z. Sun, R. Karppi, The application of electron beam welding for the joining of dissimilar metals: An overview, J. Mater. Process. Technol. 59 (3 SPEC. ISS.) (1996) 257-267.

[12] D. Herzog, V. Seyda, E. Wycisk, C. Emmelmann, Additive manufacturing of metals, Acta Mater. 117 (2016) 371-392.

[13] C. Körner, Additive manufacturing of metallic components by selective electron beam melting - a review, Int. Mater. Rev. 61 (5) (2016) 361-377.

[14] D. D. Gu, W. Meiners, K. Wissenbach, R. Poprawe, Laser additive manufacturing of metallic components: Materials, processes and mechanisms, Int. Mater. Rev. 57 (3) (2012) 133-164.

[15] W. E. Frazier, Metal additive manufacturing: A review (apr 2014).

[16] D. Ding, Z. Pan, D. Cuiuri, H. Li, Wire-feed additive manufacturing of metal components: technologies, developments and future interests (oct 2015).

[17] M. Yakout, M. A. Elbestawi, S. C. Veldhuis, A study of thermal expansion coefficients and microstructure during selective laser melting of Invar 36 and stainless steel 316L, Addit. Manuf. 24 (2018) 405-418.

[18] T. DebRoy, H. L. Wei, J. S. Zuback, T. Mukherjee, J. W. Elmer, J. O. Milewski, A. M. Beese, A. Wilson-Heid, A. De, W. Zhang, Additive manufacturing of metallic components - Process, structure and properties (mar 2018).

[19] J. J. Lewandowski, M. Seifi, Metal Additive Manufacturing: A Review of Mechanical Properties, Annu. Rev. Mater. Res. 46 (1) (2016) 151-186.

[20] J. J. Sobczak, L. Drenchev, Metallic functionally graded materials: A specific class of advanced composites, J. Mater. Sci. Technol. 29 (4) (2013) 297-316.

[21] C. Jang, J. Lee, J. Sung Kim, T. Eun Jin, Mechanical property variation within Inconel 82/182 dissimilar metal weld between low alloy steel and 316 stainless steel, Int. J. Press. Vessel. Pip. 85 (9) (2008) 635-646.

[22] T. Mukherjee, W. Zhang, T. DebRoy, An improved prediction of residual stresses and distortion in additive manufacturing, Comput. Mater. Sci. 126 (2017) 360-372.

[23] R. Banerjee, P. C. Collins, D. Bhattacharyya, S. Banerjee, H. L. Fraser, Microstructural evolution in laser deposited compositionally graded $\alpha / \beta$ titanium-vanadium alloys, Acta Mater. 51 (11) (2003) 3277-3292.

[24] P. C. Collins, R. Banerjee, S. Banerjee, H. L. Fraser, Laser deposition of compositionally graded titaniumvanadium and titanium-molybdenum alloys, Mater. Sci. Eng. A 352 (1-2) (2003) 118-128.

[25] D. C. Hofmann, S. Roberts, R. Otis, J. Kolodziejska, R. P. Dillon, J. O. Suh, A. A. Shapiro, Z. K. Liu, J. P. Borgonia, Developing gradient metal alloys through radial deposition additive manufacturing, Sci. Rep. 4 (1) (2015) 1-8.

[26] H. Sahasrabudhe, R. Harrison, C. Carpenter, A. Bandyopadhyay, Stainless steel to titanium bimetallic structure using LENS ${ }^{\text {TM }}$, Addit. Manuf. 5 (2015) 1-8.

[27] S. Q. Wang, J. H. Liu, D. L. Chen, Tensile and fatigue properties of electron beam welded dissimilar joints between Ti-6Al-4V and BT9 titanium alloys, Mater. Sci. Eng. A 584 (2013) 47-56.
[28] B. Onuike, B. Heer, A. Bandyopadhyay, Additive manufacturing of Inconel 718 - Copper alloy bimetallic structure using laser engineered net shaping (LENS ${ }^{T M}$ ), Addit. Manuf. 21 (2018) 133-140.

[29] V. K. Balla, P. D. DeVasConCellos, W. Xue, S. Bose, A. Bandyopadhyay, Fabrication of compositionally and structurally graded $\mathrm{Ti}-\mathrm{TiO} 2$ structures using laser engineered net shaping (LENS), Acta Biomater. 5 (5) (2009) 1831-1837.

[30] V. K. Balla, P. P. Bandyopadhyay, S. Bose, A. Bandyopadhyay, Compositionally graded yttria-stabilized zirconia coating on stainless steel using laser engineered net shaping (LENS $\left.{ }^{T M}\right)$, Scr. Mater. 57 (9) (2007) 861-864.

[31] G. A. Knorovsky, M. J. Cieslak, T. J. Headley, A. D. Romig, W. F. Hammetter, INCONEL 718: A solidification diagram, Metall. Trans. A 20 (10) (1989) 2149-2158.

[32] N. Nadammal, S. Cabeza, T. Mishurova, T. Thiede, A. Kromm, C. Seyfert, L. Farahbod, C. Haberland, J. A. Schneider, P. D. Portella, G. Bruno, Effect of hatch length on the development of microstructure, texture and residual stresses in selective laser melted superalloy Inconel 718, Mater. Des. 134 (2017) 139-150.

[33] S. J. Wolff, Z. Gan, S. Lin, J. L. Bennett, W. Yan, G. Hyatt, K. F. Ehmann, G. J. Wagner, W. K. Liu, J. Cao, Experimentally validated predictions of thermal history and microhardness in laser-deposited Inconel 718 on carbon steel, Addit. Manuf. 27 (2019) 540-551.

[34] K. Shah, I. ul Haq, A. Khan, S. A. Shah, M. Khan, A. J. Pinkerton, Parametric study of development of Inconelsteel functionally graded materials by laser direct metal deposition, Mater. Des. 54 (2014) 531-538.

[35] B. E. Carroll, R. A. Otis, J. P. Borgonia, J. O. Suh, R. P. Dillon, A. A. Shapiro, D. C. Hofmann, Z. K. Liu, A. M. Beese, Functionally graded material of 304L stainless steel and inconel 625 fabricated by directed energy deposition: Characterization and thermodynamic modeling, Acta Mater. 108 (2016) 46-54.

[36] A. Hinojos, J. Mireles, A. Reichardt, P. Frigola, P. Hosemann, L. E. Murr, R. B. Wicker, Joining of Inconel 718 and 316 Stainless Steel using electron beam melting additive manufacturing technology, Mater. Des. 94 (2016) 17-27.

[37] W. Oliver, G. M. Pharr, An improved technique for determining hardness and elastic modulus using load and displacement sensing indentation experiments, J. Mater. Res. 7 (6) (1992) 1564-1583.

[38] J. Kieffer, D. Karkoulis, PyFAI, a versatile library for azimuthal regrouping, J. Phys. Conf. Ser. 425 (20) (2013) 202012.

[39] S. Timoshenko, Analysis of Bi-Metal Thermostats, J. Opt. Soc. Am. 11 (3) (1925) 233.

[40] S. K. Rai, A. Kumar, V. Shankar, T. Jayakumar, K. B. S. Rao, B. Raj, Characterization of microstructures in Inconel 625 using X-ray diffraction peak broadening and lattice parameter measurements, Scr. Mater. 51 (1) (2004) 59-63.

[41] C. Yan, L. Hao, A. Hussein, P. Young, D. Raymont, Advanced lightweight 316L stainless steel cellular lattice structures fabricated via selective laser melting, Mater. Des. 55 (2014) 533-541.

[42] C. Li, Z. Y. Liu, X. Y. Fang, Y. B. Guo, Residual Stress in Metal Additive Manufacturing, in: Procedia CIRP, Vol. 71, Elsevier B.V., 2018, pp. 348-353. 
[43] D. Seo, M. Sayar, K. Ogawa, $\mathrm{SiO}_{2}$ and $\mathrm{MoSi}_{2}$ formation on Inconel 625 surface via $\mathrm{SiC}$ coating deposited by cold spray, Surf. Coatings Technol. 206 (11-12) (2012) 28512858.

[44] M. Yakout, M. A. Elbestawi, S. C. Veldhuis, A study of thermal expansion coefficients and microstructure during selective laser melting of Invar 36 and stainless steel 316L, Addit. Manuf. 24 (2018) 405-418.

[45] P. Van Houtte, L. De Buyser, The influence of crystallographic texture on diffraction measurements of residual stress, Acta Metall. Mater. 41 (2) (1993) 323-336.

[46] Z. Wang, E. Denlinger, P. Michaleris, A. D. Stoica, D. Ma, A. M. Beese, Residual stress mapping in Inconel 625 fabricated through additive manufacturing: Method for neutron diffraction measurements to validate thermomechanical model predictions, Mater. Des. 113 (2017) 169-177.

[47] P. Mercelis, J. P. Kruth, Residual stresses in selective laser sintering and selective laser melting, Rapid Prototyp. J. 12 (5) (2006) 254-265.

[48] T. Dai, J. Lippold, Characterization of the Interface of an Alloy 625 Overlay on Steels Using Nanoindentation, J. Mater. Eng. Perform. 27 (7) (2018) 3411-3418.

[49] C. Tromas, J. C. Stinville, C. Templier, P. Villechaise, Hardness and elastic modulus gradients in plasmanitrided 316L polycrystalline stainless steel investigated by nanoindentation tomography, Acta Mater. 60 (5) (2012) 1965-1973.

[50] T. Jonsson, S. Karlsson, H. Hooshyar, M. Sattari, J. Liske, J. E. Svensson, L. G. Johansson, Oxidation After Breakdown of the Chromium-Rich Scale on Stainless Steels at High Temperature: Internal Oxidation, Oxid. Met. 85 (5-6) (2016) 509-536.

[51] O. Kolednik, J. Predan, F. D. Fischer, P. Fratzl, Bioinspired Design Criteria for Damage-Resistant Materials with Periodically Varying Microstructure, Adv. Funct. Mater. 21 (19) (2011) 3634-3641.

[52] P. Fratzl, H. Gupta, F. Fischer, O. Kolednik, Hindered Crack Propagation in Materials with Periodically Varying Young's Modulus - Lessons from Biological Materials, Adv. Mater. 19 (18) (2007) 2657-2661.

[53] J. DuPont, J. Lippold, S. Kiser, Welding Metallurgy and Weldability of Nickel-Base Alloys, John Wiley \& Sons, 2009.

[54] R. A. Patterson, J. O. Milewski, GTA weld cracking-alloy 625 to 304L, Weld. J. (Miami, Fla) 64 (1985) 227-231.

[55] F. Barthelat, C. M. Li, C. Comi, H. D. Espinosa, Mechanical properties of nacre constituents and their impact on mechanical performance, J. Mater. Res. 21 (8) (2006) 1977-1986.

[56] U. G. Wegst, H. Bai, E. Saiz, A. P. Tomsia, R. O. Ritchie, Bioinspired structural materials, Nat. Mater. 14 (1) (2015) 23-36. 